Rabaska

Revue d'ethnologie de l'Amérique française

\title{
Groupe de recherches en études franco-terreneuviennes (GREF) (Memorial University)
}

\section{Scott Jamieson}

Volume 4, 2006

URI : https://id.erudit.org/iderudit/201798ar

DOI : https://doi.org/10.7202/201798ar

Aller au sommaire du numéro

Éditeur(s)

Société québécoise d'ethnologie

ISSN

1703-7433 (imprimé)

1916-7350 (numérique)

Découvrir la revue

Citer ce document

Jamieson, S. (2006). Groupe de recherches en études franco-terreneuviennes (GREF) (Memorial University). Rabaska, 4, 218-219.

https://doi.org/10.7202/201798ar d'utilisation que vous pouvez consulter en ligne.

https://apropos.erudit.org/fr/usagers/politique-dutilisation/ 


\section{Groupe de recherches en} études franco-terreneuviennes (GREF)

Département d'études françaises et hispaniques

Memorial University of Newfoundland

Courriel : scotti@mun.ca Saint-Jean (Terre-Neuve) A1B 3X8

Le Groupe de recherches en études franco-terreneuviennes (GrEF) a vu le jour en 2002 à Saint-Jean, Terre-Neuve ; depuis, cette association interdisciplinaire et bilingue informelle du département d'Études françaises et hispaniques de l'université Memorial de Terre-Neuve réunit des chercheurs (universitaires, étudiants et amateurs de différentes disciplines : Études françaises, Histoire, Études des traditions populaires, Linguistique, Anglais, Géographie, Sociologie, Sciences politiques, etc.) qui travaillent sur des aspects de la présence française à Terre-Neuve afin de partager les fruits de leurs recherches avec les membres du groupe, avec des spécialistes dans d'autres universités, avec la communauté française, et avec le public de TerreNeuve et du Labrador et d'ailleurs.

\section{Les activités du GREF 20042006}

On a mis en place un site électronique www.mun.ca/gref/indexf.html qui comporte des liens à des pages pertinentes (Patrimoine de Terre-Neuve et du Labrador, les photos de Michael Wilkshire, les pages de Patrice Brasseur à l'Université d'Avignon, les organismes francophones locaux et provinciaux, les pages sur Terre-Neuve du site Franco.ca, des sites gouvernementaux, etc.), et qui donne accès à une bibliographie, à des documents et des études préparés par les membres du Groupe.

Des séminaires ouverts au public ont lieu une ou deux fois par trimestre : conférences, tables rondes, exposés par des représentants de la communauté 
francophone et par des spécialistes d'autres universités, etc. Depuis deux ans, des chercheurs ont présenté les travaux suivants :

Amanda Crompton, «Underneath Placentia: Archaeological Explorations of the French Colony of Plaisance (1662-1713)»;

Françoise Enguehard, « 2004 Les événements commémoratifs de 500 ans de présence française à Terre-Neuve »;

Ronald Labelle, "Sauver la langue, sauver les traditions ?";

Robert Hollett \& W.J. Kirwan, « Place Names in Newfoundland : The French Names of the Northern Peninsula »;

James Hiller, " An overview of a work in progress on the history of Le Petit Nord, the area extending from Cape St. John to Cape Norman »; et un "Colloque sur l'Entente cordiale et Terre-Neuve »;

Scott Jamieson, « Julien Thoulet et son voyage à Terre-Neuve en 1886 »;

Olaf Janzen, «The Decline of France in the Newfoundland Fishery in the Eighteenth Century »;

David Quinton, « Traces. A Documentary search for the last threads of the French culture on the fishing coast of Newfoundland known as Le Petit Nord ";

Peter Pope, "Towards an Historical Archaeology of the Breton and Norman Fishery on the Petit Nord, 1500-1900»;

Ronald Rompkey, « Features of Nineteenth-century French Travel literature pertaining to Newfoundland " à partir de son livre Terre-Neuve : anthologie des voyageurs français 1814-1914.

SCOTT JAMIESON 